\title{
Genetic Symbiosis Algorithm for Multiobjective Optimization Problems ${ }^{\dagger}$
}

\author{
Jiangming MAO*, Kotaro HiRASAWA*, \\ Jinglu Hu* and Junichi MuRATA*
}

\begin{abstract}
Evolutionary Algorithms are often well-suited for optimization problems. Since mid 1980's, the interest in multi-objective problems has been expanding rapidly. Various evolutionary algorithms for multiobjective problems have been developed which are capable of searching for multiple solutions concurrently in a single run. In this paper, we propose a new genetic symbiosis algorithm (GSA) for multiobjective optimization problems (MOP) based on the symbiotic concept found widely in ecosystems. In the proposed GSA for MOP, a set of symbiotic parameters are introduced to modify the fitness of individuals used for reproduction so as to obtain a variety of Pareto solutions corresponding to user's demands. The symbiotic parameters are trained by minimizing a user defined criterion function. Several numerical simulations are carried out to demonstrate the effectiveness of the proposed GSA.
\end{abstract}

Key Words: multiobjective problems, Pareto solution, symbiosis, genetic symbiosis algorithm, evolutionary algorithm, random search, fuzzy

\section{Introduction}

Many real world problems involve simultaneous optimization of several incommensurable and often competing objectives. In these cases, there is often no single optimal solution, but rather a set of alternative solutions. These solutions are optimal in the wider sense that no other solutions in the search space are superior to them when all objectives are considered. They are known as Pareto optimal solutions. A tool exploring the design space for Pareto optimal solutions is very useful to aid the designer in arriving at a final design.

Since mid 1980's, there has been a growing interest in solving multicriteria optimization problems using evolutionary approaches. Several algorithms are available which are capable of searching for multiple Pareto optimal solutions concurrently in a single run. One of the typical methods for multiobjective problems is VEGA ${ }^{1)}$ (vector evaluated genetic algorithms) proposed by J.D. Schaffer. VEGA is an natural extension of simple genetic algorithms (SGA) in the sense that the individuals are divided and reproduced independently according to each objective function. Using VEGA, it is difficult to

\footnotetext{
$\dagger$ Proceedings of SICE Symposium on Systems and Information $2000(2000 \cdot 11)$

* Department of Electrical and Electronic Systems Engineering, Kyushu University, Fukuoka

(Received December 27, 2000)

(Revised June 11, 2001)
}

obtain equally distributed solutions in the Pareto space. In order to overcome this problem, Fonseca and Fleming ${ }^{2)}$ proposed MOGAs (multiple objective genetic algorithm) based on the ranking selection using the concept of dominated and non-dominated solutions. In addition, there have been developed many other multiobjective evolutionary methods such as NPGA ${ }^{3}$ ) (niched Pareto genetic algorithms), NSGA ${ }^{4)}$ (nondominated sorting genetic algorithm). But, these methods still have the problem, that is, the distribution of the solutions in the Pareto space can not be controlled easily by user's requests. In other words, we can not obtain the solutions in the Pareto space as we like. In order to get a variety of Pareto optimal solutions, let us pay attention to ecosystems which hold a very wide diversity. Every species seek their habitants called niche by adapting themselves to the ever changing environments. And in the niche they interact with each other by competing, exploiting and benefiting. These relations are generally called symbiosis. In this paper, we present a genetic algorithm (GA) for multiobjective optimization problems, in which a Genetic Symbiosis Algorithm (GSA) ${ }^{5)}$ and Pareto based ranking method ${ }^{2)}$ are adopted and it searches for a variety of Pareto optimal solutions based on the symbiotic concept found in the ecosystems.

GSA has been developed to obtain many kinds of solutions for optimization problems, which are optimal in one case or suboptimal in another case depending on the user's 
Table 1 Relation between symbiotic parameters in GSA and symbiotic relations in ecosystems

\begin{tabular}{|c|c|c|l|}
\hline relation & $\theta_{i j}$ & $\theta_{j i}$ & \\
\hline \hline competition & - & - & $\begin{array}{l}\text { individual } i \text { and } j \\
\text { compete each other }\end{array}$ \\
\hline predation & - & + & $\begin{array}{l}\text { individual } i \text { exploits } \\
\text { individual } j\end{array}$ \\
\hline altruism & + & - & $\begin{array}{l}\text { individual } j \text { exploits } \\
\text { individual } i\end{array}$ \\
\hline mutualism & + & + & $\begin{array}{l}\text { individual } i \text { and } j \\
\text { benefit each other }\end{array}$ \\
\hline $\begin{array}{c}\text { one side } \\
\text { damage }\end{array}$ & - & 0 & $\begin{array}{l}\text { individual } i \text { damages } \\
\text { individual } j\end{array}$ \\
\hline $\begin{array}{c}\text { one side } \\
\text { benefiting }\end{array}$ & + & 0 & $\begin{array}{l}\text { individual } i \text { benefits } \\
\text { individual } j\end{array}$ \\
\hline $\begin{array}{c}\text { neutral } \\
\text { nendion between }\end{array}$ \\
\hline
\end{tabular}

concentrative optimization problems but also multiobjective optimization problems, where many kinds of Pareto optimal solutions can be obtained according to the user's requirements.

This paper is organized as follows. Next section is a brief review on GSA. Section 3 introduces key concepts used in the field of evolutionary multicriteria optimization. Basic idea of using the concept of symbiosis to modify the fitness is described in section 4 . Section 5 states symbiotic parameters and their learning, and section 6 is devoted to summarize the training method RasID ${ }^{7)}$ mainly used for training symbiotic parameters. Section 7 gives the simulation results of some general multiobjective problems. The last section offers concluding remarks and future perspectives.

\section{Structure of Genetic Symbiosis Algo- rithm ${ }^{5)}$}

In Fig. 1 the outline of GSA is described. In GSA, reproduction is done as in the ordinary GA after diversification operation such as mutation and crossover.

GSA has two different features from the commonly used GA. The first one is that symbiotic parameters are introduced to modify the fitness of each individual. The symbiotic parameter represents the symbiotic relations such as competition, predation, altruism and mutualism between the individuals (see Table.1). Therefore, the fitness of the individual is modified depending on the symbiotic relations between the individuals. In GSA, symbiotic parameters are assumed to be calculated by fuzzy inference rules. The second feature of GSA is that it can train membership function parameters (MF parameters) constructing symbiotic relations in order to meet the designer's requirements such as diversification of the solutions. Therefore, Genetic Symbiosis Algorithm is structured in such a

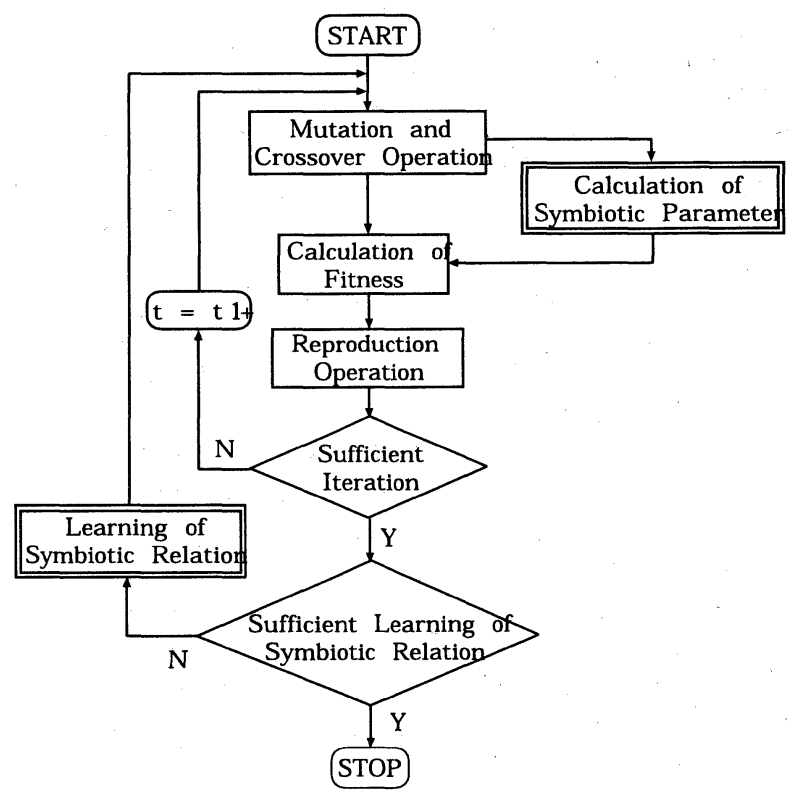

Fig. 1 Structure of Genetic Symbiosis Algorithm

way that there is an outer loop for training symbiotic relations besides the GA loop which includes the calculation of symbiotic parameters. In other words, GSA is almost the same as the commonly used GA except the use of symbiotic parameters to modify the fitness of each individual and training of MF parameters for calculating symbiotic parameters.

Fitness modification $\left(f\left(\mathbf{x}_{j}\right) \rightarrow f^{s}\left(\mathbf{x}_{j}\right)\right)$ and calculation of symbiotic parameter $\theta_{i j}(t)$ are carried out as follows. The interactions among individuals in GSA are described by symbiotic parameter $\theta_{i j}(t)$. If $\theta_{i j}(t)$ is greater than zero, then the fitness of individual $j$ is increased by the benefit from individual $i$, on the contrary, if $\theta_{i j}(t)$ is less than zero, then the fitness of individual $j$ is decreased by the damage from individual $i$. So, symbiotic parameter $\theta_{i j}(t)$ in GSA can represent the symbiotic relations in the ecosystems as shown in Table 1

$$
\begin{aligned}
f^{s}\left(\mathbf{x}_{j}\right) & \leftarrow\left(1+\sum_{i \in N} \theta_{i j}(t)\right) f\left(\mathbf{x}_{j}\right) \\
\theta_{i j}(t) & =F I\left(r_{i j}, e_{i j}, \mu_{*}, \sigma_{*}\right) \\
r_{i j} & =\sqrt{\sum_{m \in M}\left(x_{i m}-x_{j m}\right)^{2}} \\
e_{i j} & =f\left(\mathbf{x}_{i}\right)-f\left(\mathbf{x}_{j}\right)
\end{aligned}
$$

where $\mathbf{x}_{j}$ is the phenotype or genotype of individual $j$, $\mathbf{x}_{j}=\left(x_{j 1}, x_{j 2}, \ldots, x_{j M}\right), f\left(\mathbf{x}_{j}\right)$ the fitness of individual $j$ before modification, $f^{s}\left(\mathbf{x}_{j}\right)$ the fitness of individual $j$ after modification, $\theta_{i j}(t)$ the symbiotic parameter from individual $i$ to individual $j, N$ the set of suffixes of individuals, 
$F I$ the fuzzy inference function, $r_{i j}$ the distance between individual $i$ and $j, e_{i j}$ the subtracted value of fitness of individual $j$ from individual $i, M$ the set of suffixes of elements of individuals, $\mu_{*}$ and $\sigma_{*}$ are the parameters of fuzzy inference.

Hereafter, sets and their density are discribed by the same notation in the paper. The unique points of GSA are the following: symbiotic parameter $\theta_{i j}(t)$ is calculated by fuzzy inference $F I$ using $r_{i j}$ and $e_{i j}$, reproduction of individuals is executed using $f^{s}\left(\mathbf{x}_{j}\right)$ instead of $f\left(\mathbf{x}_{j}\right)$ and also the parameters $\mu_{*}$ and $\sigma_{*}$ in the fuzzy inference are trained to distribute the individuals as requested by the designer.

The reason for using fuzzy inference for calculating symbiotic parameter $\theta_{i j}(t)$ is that a priori information on symbiotic phenomena is easily installed into the fuzzy inference. As a result, the symbiotic relation like "if individual $i$ exists near individual $j$ and the fitness of individual $i$ is greater than that of individual $j$, then individual $i$ exploits individual $j "$, can be taken into account to calculate the symbiotic parameter $\theta_{i j}(t)$. Training of parameters in the fuzzy inference leads to obtaining the required distribution of the individuals.

\section{Multiobjective Problems}

\section{1 Definitions}

A general multiobjective problem is formulated by

$$
\begin{aligned}
\min \mathbf{f}(\mathbf{x}) & =\left(f_{1}(\mathbf{x}), f_{2}(\mathbf{x}), \ldots, f_{L}(\mathbf{x})\right) \\
\text { subject to } \quad \mathbf{x} & =\left(x_{1}, x_{2}, \ldots, x_{M}\right) \in X
\end{aligned}
$$

where $\mathbf{f}(\mathbf{x})$ is multiobjective function vector, $f_{l}(x)$ is $l$ th objective function, $\mathbf{x}$ is input vector, $L$ is the set of suffixes of objective functions, $M$ is the set of suffixes of elements of individuals.

\section{2 Pareto Optimality}

The scalar concept of optimality does not apply directly to the multiobjective problems. A useful replacement is the notion of Pareto optimality. Essentially, an input vector $\mathbf{x}^{*} \in X$ is said to be Pareto optimal for (MOP), if all other vectors $\mathrm{x} \in X$ have a higher value than $\mathrm{x}^{*}$ for at least one of the objective functions $f_{l}(*)$, or else have the same value for all objectives. Formally speaking, we have the following definition:

A point $\mathbf{x}^{*} \in X$ is said to be (globally) Pareto optimal or a (globally) efficient solution or a non-dominated or a non-inferior point for (MOP), if and only if there is no $\mathbf{x} \in X$ such that $f_{l}(\mathbf{x}) \leq f_{l}\left(\mathbf{x}^{*}\right)$ for all $l \in L$, with at least one strict inequality.

\section{3 Multiobjective Ranking Methods}

In the genetic algorithm with a single objective problem the fitter members of the population have higher chance of producing more offspring than the less fitter members. However, in the case of multiobjective problems, every solutions have a number of fitness values. This presents a problem in judging the overall fitness of the solutions.

Recently, most researchers of genetic algorithm have used Pareto based ranking method proposed in the book ${ }^{8)}$. In addition, as mentioned in introduction, it is now recognized that MOGAs ${ }^{2)}, \mathrm{NPGA}^{3)}$ and NSGA ${ }^{4)}$ are the useful evolutionary computational methods for multiobjective problems. However, the solutions in the Pareto space required by the designer can not be searched easily by these methods because of the following two factors, selection pressure and genetic drift. In the next subsection, we give some explanation about the effect of selection pressure and genetic drift in solving multi criteria optimization problems.

\section{4 Convergence of Population}

Alex Rogers and Adam Prügel-Bennett ${ }^{9)}$ have pointed out that the convergence of population is due to two factors. One factor is selection pressure producing multiple copies of fitter individuals while the other factor is independent of the fitness of the individuals and is due to the stochastic nature of the genetic drift. In other words, the selection pressure is produced by the optimal selection making the population converge optimally; the genetic drift is produced by random selection making the population converge randomly.

For various evolutionary algorithms for MOP, we can analyze the selection pressure and genetic drift in two cases. One is when not all the points $\mathbf{x}$ are Pareto optimal points and the other is when all points $\mathbf{x}$ are Pareto optimal points. In the first case, the selection pressure should make the population change into the second case because the second case is the target of the optimal selection. Let us pay attention to the second case where all points are non-dominated or non-inferior, in this case, each point has the same chance of producing offspring and we can consider the offspring of them are selected randomly. If we eliminate the effects of mutation and crossover, we can see the effects of genetic drift, making the population converge randomly. The proposed Genetic Symbiosis Algorithm for multiobjective problems could be considered as a method to avoid the above convergence by the genetic drift and to distribute the individuals as requested by the users demand using the symbiotic concept. 


\section{Basic Structure of GSA for MOP ${ }^{6)}$}

As presented in the previous section, all Pareto solutions are non-dominated, so the selection pressure is equal to zero in the second case. However by paying attention to ecosystems, we can find that the selection pressure can be produced by not only some environmental conditions such as temperature, water and food, but also some interactions among species. So in this paper, the symbiotic parameter $\theta_{l i j}$ and $\theta_{m l}$ similar to $\theta_{i j}$ in section 2 are introduced. The symbiotic parameter $\theta_{l i j}$ represents the symbiotic relations regarding $l$ th objective function, such as competition, predation, altruism and mutualism between the individuals, while the symbiotic parameter $\theta_{m l}$ describes the interactions among objective functions. These parameters can change the selection pressure and hold Pareto solutions in a wide diversity as requested by the designers.

As shown in Fig.1, GSA for MOP is structured in such a way that there is an outer loop for training symbiotic relations besides the main GA loop which includes the calculation of symbiotic parameters.

In the Main GA loop, we use symbiotic parameters $\theta_{l i j}$ and $\theta_{m l}$ to modify the fitness and calculate the rank of each individual. Rank is calculated as well as fitness by symbiotic parameter $\theta_{l i j}$ and $\theta_{m l}$ in GSA for MOP in stead of conventional GSA. Reproduction is done as in the ordinary GA after diversification processing such as mutation and crossover. In the outer loop, the symbiotic relations are trained by using a modified random search method called $\operatorname{RasID}^{7)}$ in the same way as conventional GSA. In this paper, optimization of real coded functions is considered.

\section{1 Ranking Population}

The rank of each individual is calculated based on the modified fitnesses of individuals. The ranking method presented in reference ${ }^{2)}$ is used in the main loop of GSA for MOP; if individual $i$ dominates $n$ other individuals, then the rank of individual $i$ is $n+1$.

\section{2 Fitness Modification}

We use symbiotic parameters $\theta_{l i j}$ and $\theta_{m l}$ to modify the fitness of individual $\mathbf{x}_{j}$ regarding $l$ th objective function as follows.

$$
f_{l}^{s}\left(\mathbf{x}_{j}\right) \leftarrow\left[1+\sum_{m \in L} \theta_{m l}\left(\mathbf{x}_{j}\right)+\sum_{i \in N} \theta_{l i j}\left(\mathbf{x}_{j}\right)\right] f_{l}\left(\mathbf{x}_{j}\right)
$$

where $\mathbf{x}_{j}$ is phenotype or genotype of individual $j, f_{l}\left(\mathbf{x}_{j}\right)$ fitness of individual $\mathbf{x}_{j}$ regarding $l$ th objective function before modification, $f_{l}^{s}\left(\mathbf{x}_{j}\right)$ fitness of individual $j$ regarding $l$ th objective function after modification, $\theta_{m l}\left(\mathbf{x}_{j}\right)$ sym- biotic parameter from $m$ th objective function to $l$ th objective function, $\theta_{l i j}\left(\mathbf{x}_{j}\right)$ symbiotic parameter from individual $i$ to individual $j$ regarding $l$ th objective function, $N$ the set of suffixes of individuals, $L$ the set of suffixes of objective functions.

\section{Symbiotic Parameters}

In this section, it is explained how symbiotic parameters $\theta_{l i j}$ and $\theta_{m l}$ for modifying the fitness can be calculated by fuzzy inference, in addition how the parameters of fuzzy inference (membership function parameters) are trained by RasID optimization method in order to satisfy the designer's demand on the distribution of the individuals.

\section{1 Symbiotic Parameter $\theta_{m l}$}

As stated in the previous section, symbiotic parameter $\theta_{m l}$ describes the interactions among objective functions. We can calculate it from the normalized distance $\bar{f}_{m l}\left(\mathbf{x}_{j}\right)$ between $f_{l}\left(\mathbf{x}_{j}\right)$ and $f_{m}\left(\mathbf{x}_{j}\right)$ for each individual $\mathbf{x}_{j}$ by using fuzzy inference as follows.

$$
\text { If } \begin{array}{r}
\bar{f}_{m l}\left(\mathbf{x}_{j}\right) \text { is } F_{q} \text { then } \theta_{m l} \text { is } \Theta_{q} \\
m, l \in L \text { and } q \in Q
\end{array}
$$

Membership functions of fuzzy set $F_{q}$ and $\Theta_{q}$ are supposed to be the following gaussian functions.

$$
\begin{aligned}
& f_{f q}\left(\bar{f}_{m l}\right)=e^{-\frac{\left(\bar{f}_{m l}-\mu_{f q}\right)^{2}}{2 \sigma_{f q}^{2}}} \\
& f_{\theta q}\left(\theta_{m l}\right)=e^{-\frac{\left(\theta_{m l}-\mu_{\theta q}\right)^{2}}{2 \sigma_{\theta q}^{2}}}
\end{aligned}
$$

where

$$
\begin{aligned}
& f_{m l}=f_{m}\left(\mathbf{x}_{j}\right)-f_{l}\left(\mathbf{x}_{j}\right) \\
& \bar{f}_{m l}=\frac{f_{m l}}{\left|f_{\max }-f_{\min }\right|} \\
& f_{\min } \leq f_{m}\left(\mathbf{x}_{j}\right), f_{l}\left(\mathbf{x}_{j}\right) \leq f_{\max } \\
& Q: \text { the set of suffixes of fuzzy rules }
\end{aligned}
$$

if part and then part of the fuzzy rules can be calculated by (8) and (9), and $\theta_{m l}$ can be calculated by (10) using the following center-of-gravity method.

$$
\theta_{m l}=\frac{\sum_{q \in Q} f_{f q}\left(\bar{f}_{m l}\right) \mu_{\theta q} \sigma_{\theta q}}{\sum_{q \in Q} f_{f q}\left(\bar{f}_{m l}\right) \sigma_{\theta q}}
$$

\section{2 Symbiotic Parameter $\theta_{l i j}$}

Since symbiotic parameters $\theta_{l i j}$ describes the interactions among individuals, we can calculate it from the normalized distance $\bar{r}_{i j}$ between individual $\mathbf{x}_{i}$ and $\mathbf{x}_{j}$ and the normalized distance $\bar{f}_{l i j}$ between $f_{l}\left(\mathbf{x}_{i}\right)$ and $f_{l}\left(\mathbf{x}_{j}\right)$ by 
using the following fuzzy inference rules.

If $\bar{r}_{i j}$ is $R_{l q}$ and $\bar{f}_{l i j}$ is $F_{l q}$ then $\theta_{l i j}$ is $\Theta_{l q}$

$$
l \in L i, j \in N \text { and } q \in Q
$$

Membership functions of fuzzy set $R_{l q}, F_{l q}$ and $\Theta_{l q}$ are assumed as follows using gaussian functions.

$$
\begin{aligned}
f_{r l q}\left(\bar{r}_{i j}\right) & =e^{-\frac{\left(\bar{r}_{i j}-\mu_{r l q}\right)^{2}}{2 \sigma_{r l q}^{2}}} \\
f_{f l q}\left(\bar{f}_{l i j}\right) & =e^{-\frac{\left(\bar{f}_{l i j}-\mu_{f l q}\right)^{2}}{2 \sigma_{f l q}^{2}}} \\
f_{\theta l q}\left(\theta_{l i j}\right) & =e^{-\frac{\left(\theta_{l i j}-\mu_{\theta l q}\right)^{2}}{2 \sigma_{\theta l q}^{2}}}
\end{aligned}
$$

where

$$
\begin{aligned}
& r_{i j}=\sqrt{\sum_{m \in M}\left(x_{i m}-x_{j m}\right)^{2}} \\
& \bar{r}_{i j}=\frac{r_{i j}}{\sqrt{M\left(x_{\max }-x_{\min }\right)^{2}}} \\
& f_{l i j}=f_{l}\left(\mathbf{x}_{i}\right)-f_{l}\left(\mathbf{x}_{j}\right) \\
& \bar{f}_{l i j}=\frac{f_{l i j}}{\left|f_{\max }-f_{\min }\right|} \\
& x_{\min } \leq x_{i m}, x_{j m} \leq x_{\max } \\
& f_{\min } \leq f_{l}\left(\mathbf{x}_{i}\right), f_{l}\left(\mathbf{x}_{j}\right) \leq f_{\max } \\
& Q: \text { the set of suffixes of fuzzy rules }
\end{aligned}
$$

$M$ : the set of suffixes of elements of individuals

Therefore symbiotic parameter $\theta_{l i j}$ can be calculated by (11) - (14) using the following center-of-gravity method as the previous subsection.

$$
\theta_{l i j}=\frac{\sum_{q \in Q}\left\{f_{r l q}\left(\bar{r}_{i j}\right) \cap f_{f l q}\left(\bar{f}_{l i j}\right)\right\} \mu_{\theta l q} \sigma_{\theta l q}}{\sum_{q \in Q}\left\{f_{r l q}\left(\bar{r}_{i j}\right) \cap f_{f l q}\left(\bar{f}_{l i j}\right)\right\} \sigma_{\theta l q}}
$$

\section{3 Training of Membership function Parame- ters}

From the previous subsection, it has become clear that symbiotic parameters can be calculated by the inference rules that have many membership function (MF) parameters like $\mu_{f q}, \sigma_{f q}, \mu_{\theta q}, \sigma_{\theta q}, \mu_{r l q}, \sigma_{r l q}, \mu_{f l q}, \sigma_{f l q}, \mu_{\theta l q}$ and $\sigma_{\theta l q}$. Learning of symbiotic relations here means that MF parameters are trained to meet the designer's requirements on the distribution of the individuals.

User's requirements can be described as a criterion function. For example, we can give a criterion function as follows.

$$
\begin{aligned}
L= & k_{R} \sum_{j \in N} R\left(\mathbf{x}_{j}\right) \\
& +k_{E f} e^{-\alpha E_{f}}+k_{V f} \sum_{j \in N}\left(D_{m f}\left(\mathbf{x}_{j}\right)-E_{f}\right)^{2}
\end{aligned}
$$

$$
+k_{E r} e^{-\beta E_{r}}+k_{V r} \sum_{j \in N}\left(D_{m r}\left(\mathbf{x}_{j}\right)-E_{r}\right)^{2}
$$

where

$$
\begin{aligned}
& D_{f}(i j)=\sqrt{\sum_{l \in L}\left(f_{l}\left(\mathbf{x}_{i}\right)-f_{l}\left(\mathbf{x}_{j}\right)\right)^{2}} \\
& D_{m f}\left(\mathbf{x}_{j}\right)=\min \left\{D_{f}(i j)\right\} \quad i \in N \\
& E_{f}=\frac{1}{N} \sum_{j \in N} D_{m f}\left(\mathbf{x}_{j}\right) \\
& D_{r}(i j)=r_{i j} \\
& D_{m r}\left(\mathbf{x}_{j}\right)=\min \left\{D_{r}(i j)\right\} \quad i \in N \\
& E_{r}=\frac{1}{N} \sum_{j \in N} D_{m r}\left(\mathbf{x}_{j}\right)
\end{aligned}
$$

where $R\left(\mathbf{x}_{j}\right)$ is the rank of individual $j$ using $f_{l}\left(\mathbf{x}_{j}\right)$, i.e., original fitness functions. $k_{R}, k_{E f}, k_{V f}, k_{E r}, k_{V r}, \alpha$ and $\beta$ are the weighting coefficients. $D_{f}(i j)$ and $D_{r}(i j)$ are the distance between individual $i$ and individual $j$ in the gnome and the fitness space: $D_{m f}\left(\mathbf{x}_{j}\right)$ and $D_{m r}\left(\mathbf{x}_{j}\right)$ are the minimum distance of individual $j$ to other individuals.

In this criterion, we can consider the individual $i$ which is the nearest to individual $j$ in the genome or the fitness space as the neighbor of individual $j$. The expectation of $D_{m f}\left(\mathbf{x}_{j}\right)$ and $D_{m r}\left(\mathbf{x}_{j}\right)$ is trained to maximize and the variance of $D_{m f}\left(\mathbf{x}_{j}\right)$ and $D_{m r}\left(\mathbf{x}_{j}\right)$ is trained to minimize. It can make the solutions well-distributed in the sense that individuals are distributed uniformly and widely in the Pareto optimal space. On the other hand, by minimizing the sum of the rank for each individual we can get Pareto solutions. Of course, the above is the most important condition to obtain Pareto optimal solutions, so we should set the weighting coefficient $k_{R}$ larger than other coefficients. The weighting coefficient $k_{E f}, k_{V f}, k_{E r}, k_{V r}$ can be changed by user's requirements. So this criterion function makes it possible to search for a variety of Pareto solutions and make them well-distributed in Pareto space as required by the designers.

Furthermore, GSA can not obtain a real optimal Pareto front because fitness $f_{l}^{s}\left(\mathbf{x}_{j}\right)$ is modified by Eq.(6). But, as is known from Eq.(6), it is supposed that the second and the third terms of right hand side of Eq.(6) are smaller than 1.0. Therefore, an approximate Pareto optimal front can be obtained by GSA using a modified fitness function like Eq.(6). The trade off between getting an optimal Pareto front and distributing individuals in the genome and fitness space as we like can be adjusted by coefficients $k_{R}, k_{E f}, k_{V f}, k_{E r}$ and $k_{V r}$ in Eq.(16). Eq.(16) can not be used directly as a criterion function because' Eq.(16) can be calculated only when all the individuals are known, in other words, the fitness of each individual can not be cal- 
culated by Eq.(16). In addition, in the proposed GSA for MOP, symbiotic parameters $\theta_{m l}$ and $\theta_{l i j}$ once obtained using Eq.(16) can be used many times whenever only an inner loop of GSA is executed. In other words, without training symbiotic parameters, the main loop of GSA for MOP can be calculated many times we want. One of the examples using this scheme will be illustrated later in Fig.4.

\section{Random Search with Intensification and Diversification (RasID) ${ }^{7)}$}

The gradient-based method is often used to train parameters, but the gradient information cannot be available in the proposed model. In this paper, we train the MF parameters by using a modified random search method called RasID (Random Search with Intensification and Diversification). Therefore, given membership function parameters $\mu_{f q}, \sigma_{f q}, \mu_{\theta q}, \sigma_{\theta q}, \mu_{r l q}, \sigma_{r l q}, \mu_{f l q}$, $\sigma_{f l q}, \mu_{\theta l q}$ and $\sigma_{\theta l q}$, we can calculate $\theta_{m l}\left(\mathbf{x}_{j}\right)$ and $\theta_{l i j}\left(\mathbf{x}_{j}\right)$ for each individual $\mathbf{x}_{j}$. Then the problem is to train membership function parameters so that the required distribution of the individuals is obtained.

RasID(Random Search with Intensification and Diversification) is a kind of random search optimization methods and executes intensified and diversified search in a unified manner using information on success and failure of the past searching. Basic idea of RasID is that it continues to iterate the searching in the following way. When there is quite a possibility of finding a better solution around the current one, intensified search is executed near the current solution, and when finding a better solution can not be expected because of falling into local minima, then diversified search is executed looking for a better solution. One of the distinguished features of RasID is that the probability density function for random searching is changed adaptively depending on the success and failure information of the past searching. So, by using RasID, gradient calculation is not needed, faster computation is expected and escaping from local minima becomes possible.

\section{Simulations}

\section{1 Simulations of a nonconvex multiobjective function}

The following very simple nonconvex function for multiobjective optimizers are used in order to demonstrate the effectiveness of the proposed method in this subsection,

$$
\begin{array}{cl}
\min & \left(f_{1}\left(x_{1}, x_{2}\right), f_{2}\left(x_{1}, x_{2}\right)\right) \\
\text { where } & f_{1}\left(x_{1}, x_{2}\right)=2 \sqrt{x_{1}}
\end{array}
$$
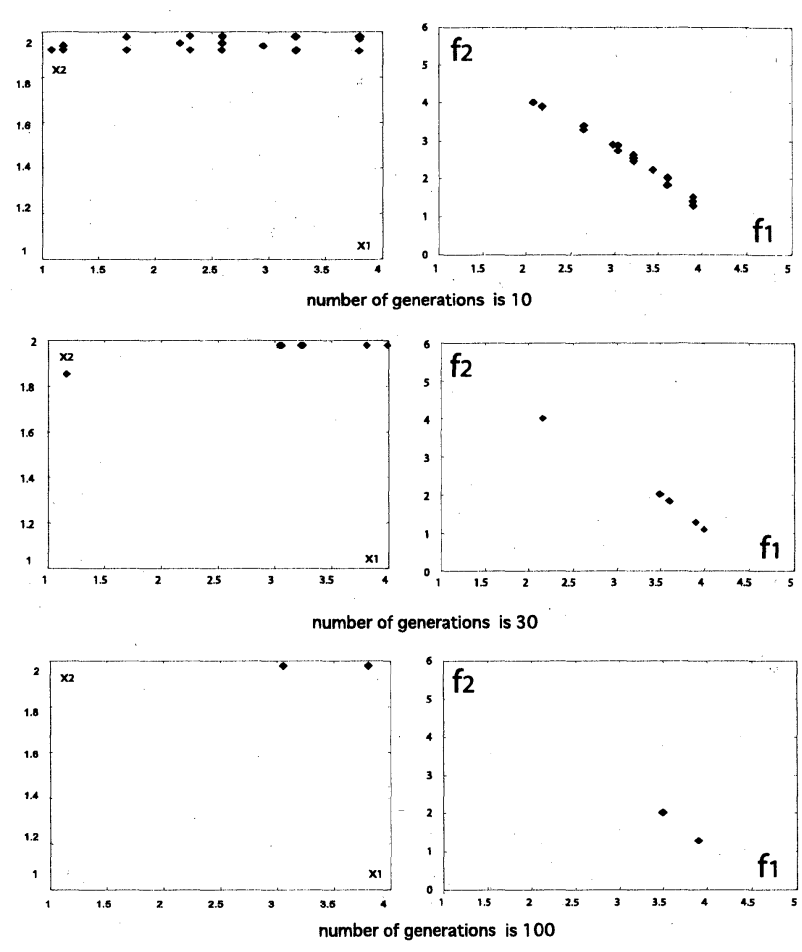

Fig. 2 Simulation results of a nonconvex multiobjective function (conventional method)

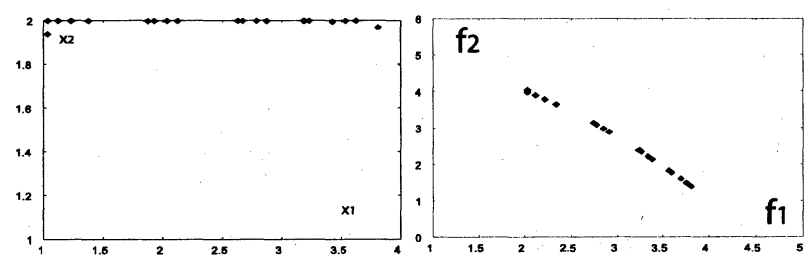

Fig. 3 Simulation results of a nonconvex multiobjective function (proposed method)

$$
\begin{aligned}
& f_{2}\left(x_{1}, x_{2}\right)=x_{1}\left(1-x_{2}\right)+5 \\
& x_{1} \in[1,4] \\
& x_{2} \in[1,2]
\end{aligned}
$$

In this simulation, the number of individuals $N$ is 40 , mutation rate is 0.01 , as for crossover, two-point crossover is used and crossover rate is 0.4. The upper part, the middle part and the lower part of Fig.2 show the results when the number of generations is equal to 10,30 and 100 , respectively by using conventional Ranking Method. We can see that the distribution of the individuals in the genome space and fitness space becomes smaller and smaller along with the increase of the number of generation.

Fig.3 shows the results by using the proposed method. In this simulation, the number of individuals $N$ is 40 , the number of generations is 100 , and the number of iterations for RasID training of MF parameters is 1000. Mutation rate is 0.01 , as for crossover, two-point crossover 
Table 2 Fuzzy parameters in Eq.(8)-(9)

\begin{tabular}{|c|c|c|l|}
\hline rule & rule 1 & rule 2 & rule 3 \\
\hline \hline$\mu_{f q}$ & -.079799 & .042619 & .056976 \\
\hline$\sigma_{f q}$ & .039187 & .001111 & .060964 \\
\hline$\mu_{\theta q}$ & .015201 & -.070656 & .036020 \\
\hline$\sigma_{\theta q}$ & .077825 & .067777 & .042962 \\
\hline
\end{tabular}

Table 3 Fuzzy parameters in Eq.(12)-(14)

\begin{tabular}{|c|c|c|c|l|}
\hline rule & rule 1 & rule 2 & rule 3 & rule 4 \\
\hline \hline$\mu_{r 1 q}$ & .020018 & -.041543 & .000734 & .034998 \\
\hline$\sigma_{r 1 q}$ & .032864 & .005139 & .046428 & .006262 \\
\hline$\mu_{f 1 q}$ & -.028744 & .009784 & .026077 & -.016944 \\
\hline$\sigma_{f 1 q}$ & .031861 & .024669 & .032714 & .100269 \\
\hline$\mu_{\theta 1 q}$ & -.026308 & -.059218 & .019830 & -.023053 \\
\hline$\sigma_{\theta 1 q}$ & .015878 & .004019 & .013742 & .045115 \\
\hline \hline$\mu_{r 2 q}$ & .043454 & .073890 & -.005512 & -.019013 \\
\hline$\sigma_{r 2 q}$ & .052837 & .028827 & .014284 & .068198 \\
\hline$\mu_{f 2 q}$ & .008170 & -.075437 & .003781 & -.108653 \\
\hline$\sigma_{f 2 q}$ & .012193 & .073835 & .043058 & .034240 \\
\hline$\mu_{\theta 2 q}$ & -.026815 & .029736 & -.062257 & .035785 \\
\hline$\sigma_{\theta 2 q}$ & .039249 & .052759 & .011022 & .061657 \\
\hline
\end{tabular}
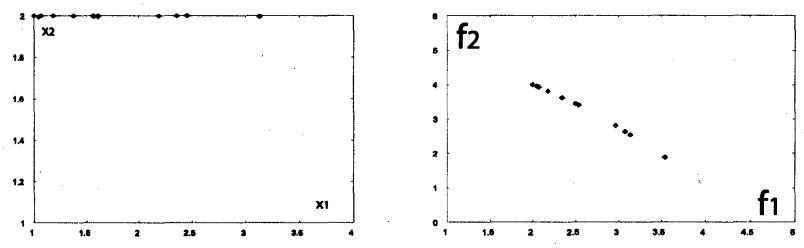

Fig. 4 Simulation results using fuzzy parameters in Table 2 and 3

is used and crossover rate is 0.4. Furthermore, $k_{R}=10$, $k_{E f}=k_{V f}=1000, k_{E r}=k_{V r}=0$ and $\alpha=100$ are used in Eq. (16).

The left part of Fig. 3 shows the distribution of the individuals in $\left(x_{1}, x_{2}\right)$ space, while fitness values of 40 individuals are plotted in $\left(f_{1}, f_{2}\right)$ space. It can be clarified from Fig. 3 that GSA for MOP is able to approximate the Pareto-optimal front well for the simple multiobjective problem.

The fuzzy parameters which were trained in this simulation are shown in Table2 and 3. From Table2 and 3 , we can not easily show how the individuals are controlled, so we did the following study. We carried out simulations using fuzzy parameters in Table2 and 3 and not using the outer loop training any more in GSA. The results are shown in Fig.4. Comparing Fig.2 and Fig.4, we can see that the problem of concentrating individuals by a conventional method can be overcome by using GSA for MOP.

\subsection{Simulations of a convex multiobjective function}

In this subsection, we studied the effectiveness of the proposed method using the following two dimensional
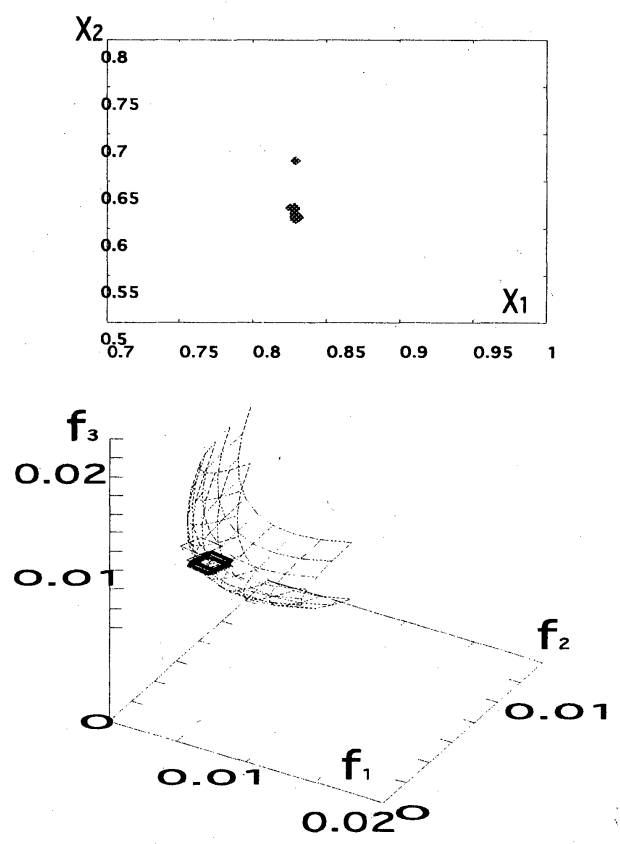

Fig. 5 Simulation results of a convex multiobjective function (conventional method)
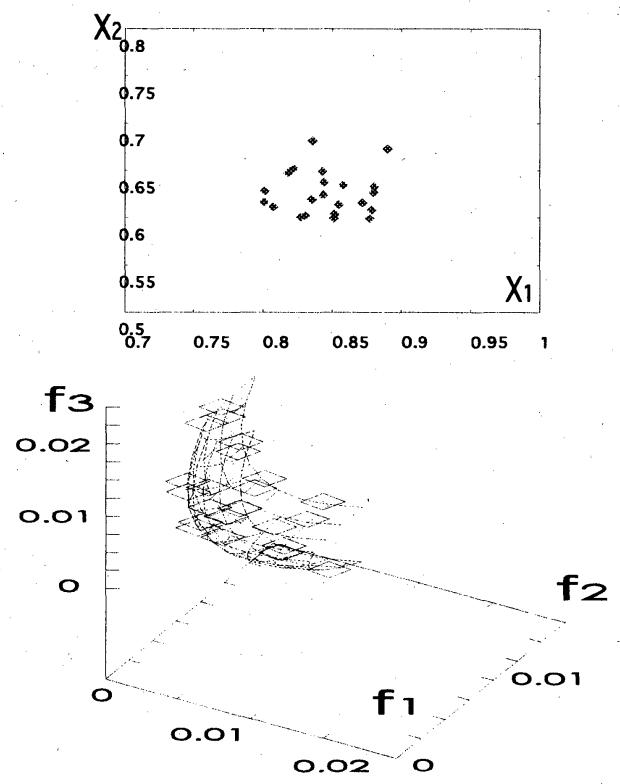

Fig. 6 Simulation results of a convex multiobjective function (proposed method)

three multiobjective functions,

$$
\begin{aligned}
\min & \left(f_{1}\left(x_{1}, x_{2}\right), f_{2}\left(x_{1}, x_{2}\right), f_{3}\left(x_{1}, x_{2}\right)\right) \\
\text { where } & f_{1}\left(x_{1}, x_{2}\right)=\left(x_{1}-0.8\right)^{2}+\left(x_{2}-0.6\right)^{2} \\
& f_{2}\left(x_{1}, x_{2}\right)=\left(x_{1}-0.85\right)^{2}+\left(x_{2}-0.7\right)^{2} \\
& f_{3}\left(x_{1}, x_{2}\right)=\left(x_{1}-0.9\right)^{2}+\left(x_{2}-0.6\right)^{2} \\
& x_{1}, x_{2} \in[0,1]
\end{aligned}
$$

In this simulation, the number of individuals $N$ is 30 , the number of generations is 500 , and the number of iterations for RasID training of MF parameters is 1000 . Mu- 

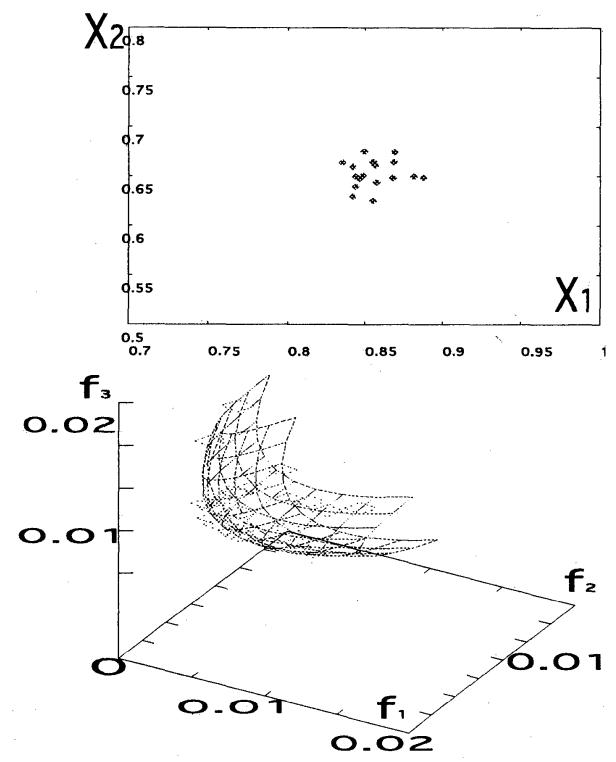

Fig. 7 Simulation result of setting the distribution of individuals to a designated value

tation rate is 0.05 , as for crossover, two-point crossover is used and crossover rate is 0.8 . Fig. 5 shows the results when the $k_{R}=1$ and $k_{E f}=k_{V f}=k_{E r}=k_{V r}=0$ are used. Therefore, it corresponds to the conventional ranking method. The upper part of the figure describes the distribution of individuals in the $\left(x_{1}, x_{2}\right)$ space, while the distribution of individuals in the $\left(f_{1}, f_{2}, f_{3}\right)$ space are described in the lower parts of the figure. It is clear from Fig. 5 that the distribution of individuals both in $\left(x_{1}, x_{2}\right)$ space and $\left(f_{1}, f_{2}, f_{3}\right)$ space is fairly concentrated when only the ranking method is used.

Then simulations are carried out when weighting coefficients are set as $k_{E f}=k_{V f}=k_{E r}=k_{V r}=0.1$ which means we expect the solutions are various and welldistributed in Pareto space. The results are shown in Fig.6. We can see that there are 22 different solutions and variance of them are considerably good. Comparing Fig.5 and Fig.6, we can see that the problem of concentrating individuals by a conventional method can be overcome by using GSA for MOP.

\subsection{Simulations of setting the distributions of individuals to a designated value}

If we want to set the distribution of individuals, i.e., to set $E_{f}$ and $E_{r}$ to special values, we can change the Eq. (16) as follows.

$$
\begin{aligned}
L= & k_{R} \sum_{j \in N} R\left(\mathbf{x}_{j}\right) \\
& +k_{E f}\left(E_{f}-E_{f o}\right)^{2}+k_{V f} \sum_{j \in N}\left(D_{m f}\left(\mathbf{x}_{j}\right)-E_{f}\right)^{2}
\end{aligned}
$$

$$
+k_{E r}\left(E_{r}-E_{r o}\right)^{2}+k_{V r} \sum_{j \in N}\left(D_{m r}\left(\mathbf{x}_{j}\right)-E_{r}\right)^{2}
$$

where $E_{f o}$ and $E_{r o}$ are the target values of $E_{f}$ and $E_{r}$.

Here, we considered only the expectation and variance in the gnome space and set weighting coefficients to $k_{E f}=k_{V f}=0, k_{E r}=k_{V r}=0.1$. The target value of $E_{r}$ was set to $E_{r o}=0.001$. Other conditions are the same as the previous simulations. The results are shown in Fig. 7. It is known from Fig. 6 and 7 that the individuals in Fig.7 are distributed in a smaller area than the individuals in Fig.6. It means that the distribution of individuals can be controlled easily by using GSA for MOP.

\section{Conclusion}

In this paper, GSA for MOP which is based on the concept of symbiosis found widely in ecosystems is presented. Distinguished points of GSA for MOP are that (1) the calculation of the ranking is executed using the modified fitness $f_{l}^{s}\left(\mathbf{x}_{j}\right)$ instead of the original fitness $f_{l}\left(\mathbf{x}_{j}\right),(2)$ symbiotic parameters are calculated by fuzzy inference, and (3) fuzzy parameters can be trained by RasID learning to realize the required distribution of individuals in the genome and the fitness space.

From the simulations, it has been clarified that the GSA for MOP is useful to realize the distribution of individuals as we like. When the symbiotic parameters $\theta_{m l}$ and $\theta_{l i j}$ are reused in the main loop of GSA for MOP, the computation cost of GSA for MOP is almost the same as conventional Ranking Method. But, current full GSA for MOP is not a fast computation method, because it uses the symbiotic parameters which simulate the symbiotic relationships found in natural ecosystems to change the selection pressure dynamically. Speeding up the computation time is one of the next topics for GSA for MOP

\section{References}

1) J.D. Schaffer: Multiple Objective Optimization with Vector Evaluated Genetic Algorithms, in Proc. of the first International Conference on Genetic Algorithms and Their Applications, 160/168 (1985)

2) C.M. Fonseca and P.J. Fleming: Genetic Algorithms for Multiobjective Optimization: Formulation, Discussion and Generalization, in Proc. of 5th Int. Conf. on Genetic Algorithms, 416/423 (1993)

3) J. Horn, N. Nafpliotis and D. E. Goldberg: A Niched Pareto Genetic Algorithm for Multiobjective Optimization, in Proc. of the First IEEE Conference on Evolutionary Computation, 82/87 (1994)

4) N. Svinivas and K. Deb: Multiobjective Optimization Using Nondominated Sorting in Genetic Algorithms, IEEE Trans. Evol. Comput., 2-3, 221/248 (1995)

5) K. Hirasawa, Y. Ishikawa, J. Hu, C. Jin and J. Murata: Genetic Symbiosis Algorithm, in Proc. Congress on Evolutionary Computation, 1377/1384, July (2000) 
6) J. Mao, K. Hirasawa, J. Hu and J. Murata: Genetic Symbiosis Algorithm for Multiobjective Optimization Problems, in Proc. the Genetic and Evolutionary Computation Conference, San Francisco, U.S.A. July (2001)

7) J. Hu, K. Hirasawa and J. Murata: RasID-A Random Search Method for Neural Networks Training, Journal of Advanced Computational Intelligence, 2-4, 134/141 (1998)

8) D.E. Goldberg: Genetic Algorithms in Search, Optimization and Machine Learning, Addison-Wesley (1989)

9) Alex Rogers, Adam Prügel-Bennett: Genetic Drift in Genetic Algorithm Selection Schemes, IEEE Trans. Evol. Comput., 3-4, 298/303, Nov. (1999)

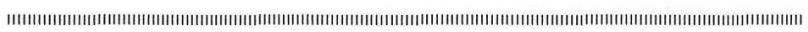

\section{Jiangming MAO}

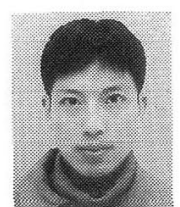

received the B.S degree from Nanjing University of Science and Technology, China, in 1995, and M.S. degree in Electrical and Electronic Systems Engineering from kyushu University, Japan, in 2001. Now he is a first-year Dr. candidate at the Graduate School of Information Science and Electrical Engineering, Kyushu University. His current research interest lies in evolutionary computation.

\section{Kotaro Hirasawa (Member)}

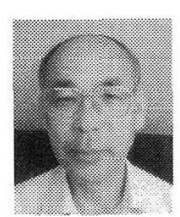

received the B.S. and M.S. degrees in electrical engineering from Kyushu University, Japan in 1964 and 1966, respectively.

From 1966 to 1992, he was with Hitachi Ltd., where he served as a Vice President at the Hitachi Research Laboratory. Since December 1992, he has been a Professor in the Faculty of Engineering, Kyushu University. He is currently with the Graduate School of Information Science and Electrical Engineering at the same university.

Dr. Hirasawa is a member of IEEE.

Jinglu Hu (Member)

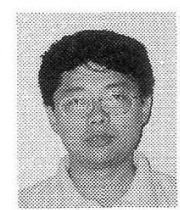

received the B.S degree and the M.S degree in electronic engineering from Zhongshan University, China, in 1983 and 1986 respectively, and the Ph.D degree in computer science and engineering from Kyushu Institute of Technology, Japan, in 1997.

From 1986 to 1993, he worked at Zhongshan University, where he was a Research Associate and then Lecturer. Since 1997, he has been a Research Associate at Graduate School of Information Science and Electrical Engineering, Kyushu University, Japan. His current research interests are system identification, learning network and their applications. $\mathrm{Dr} \mathrm{Hu}$ is a member of the Society of Instrument and Control Engineers, a member of the Institute of Electrical Engineers of Japan.

\section{Junichi Murata (Member)}

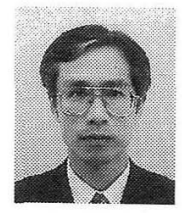

received the Master and Doctor of Engineering degrees from Kyushu University, Japan, in 1983 and 1986, respectively.

He then became a Research Associate and an Associate Professor on the Faculty of Engineering, Kyushu University. $\mathrm{He}$ is currently an Associate Professor at Graduate School of Information Science and Electrical Engineering. Kyushu University. His current research interests are neural networks, self-organizing systems and their applications to control and identification.

Dr. Murata is a member of SICE, ISCIE, IEEJ and IEEE. 